Décadrages Décadrages

cinéma, à travers champs Cinéma, à travers champs

29-30 | 2015

René Vautier

\title{
Les films antinucléaires de René Vautier
}

\section{Sylvain Portmann}

\section{(2) OpenEdition}

Journals

Édition électronique

URL : https://journals.openedition.org/decadrages/803

DOI : $10.4000 /$ decadrages. 803

ISSN : 2297-5977

\section{Éditeur}

Association Décadrages

\section{Édition imprimée}

Date de publication : 1 avril 2015

Pagination : 142-151

ISBN : 9782970096306

ISSN : 2235-7823

\section{Référence électronique}

Sylvain Portmann, «Les films antinucléaires de René Vautier », Décadrages [En ligne], 29-30 | 2015, mis en ligne le 01 mars 2017, consulté le 26 mars 2023. URL : http://journals.openedition.org/decadrages/ 803 ; DOI : https://doi.org/10.4000/decadrages.803

\section{(c) $(1)(9)$}

Creative Commons - Attribution - Pas d'Utilisation Commerciale - Partage dans les Mêmes Conditions 4.0 International - CC BY-NC-SA 4.0

https://creativecommons.org/licenses/by-nc-sa/4.0/ 
Sylvain Portmann

\section{Les films antinucléaires de René Vautier}

L'édition récente par la coopérative des Mutins de Pangée d'une série de films réalisés par René Vautier a permis de donner une nouvelle visibilité à bon nombre d'œuvres difficilement accessibles. On y trouve ainsi les célèbres Afrique 50 ou Avoir 20 ans dans les Aurès, tout comme un ensemble de films en lien avec l'Algérie, dont certains sont abordés dans ce dossier. La Cinémathèque de Bretagne, dépositaire des films, en conserve pourtant un nombre important qui ne figure pas au sommaire de ces rééditions. Parmi ceux-ci, nous en avons retenu trois qui forment un ensemble thématique autour de la question du nucléaire: Paris pour la paix (coréalisé avec Gérard Binse, France, 1986), Mission Pacifique (coréalisé avec Michel Le Thomas, F., 1988) et Hirochirac 1995 (F., 1996). Nous proposons ici de revenir sur leur importance historique, politique et sociale. Car cet ensemble dévoile des données chiffrées (nombre et dates d'essais nucléaires, de bombardements, etc.), les astuces politiciennes ayant trait à la dissimulation des dommages ou des dangers qui en découlent et finalement, comme souvent chez Vautier, un appel à la prise de conscience et à la responsabilité auprès du spectateur: ce dernier est invité à réagir face aux dangers de l'ignorance et de la passivité citoyenne et à s'opposer aux agissements inacceptables de certains gouvernements (à commencer par le sien, le français).

La distribution restreinte de ces films - due, entre autres, à diverses interdictions et peut-être à leur production de type télévisuel - semble être à l'origine de l'absence de toute couverture médiatique ou plus largement de leur méconnaissance. Ironiquement, et c'est René Vautier

1 Signalons que l'ouvrage Caméra $\mathrm{C} i$ toyenne. Mémoires (Rennes, Apogée, I998), épuisé depuis plusieurs années, devrait être prochainement réédité. qui le suggère dans ses mémoires ${ }^{1}$, la production de films et la censure (parfois indirecte) peuvent être intrinsèquement liées, pour autant que l'on s'attache aux mécanismes de diffusion; revenant sur une visite qu'un haut fonctionnaire de l'Etat français lui avait faite en janvier 1973 alors qu'il était alité en raison d'une grève de la faim - menée afin d'exiger «[la] suppression de la possibilité, pour la commission de censure cinémato- 
graphique, de censurer des films sans fournir de raisons; et l'interdiction, pour cette commission, de demander des coupes ou refus de visa pour des critères politiques $»^{2}-$, Vautier transcrit ceci, du discours qu'on lui avait tenu:

«Votre but est d'obtenir la suppression de la censure politique sur le cinéma français? Vous obtiendrez probablement satisfaction: le ministre suggérera à la commission de censure de ne plus demander de coupes ou d'interdiction pour des raisons politiques [...]. Mais, si vous espérez que cela libérera l'expression de vos convictions politiques par le film, vous vous trompez complètement, car il est bien évident que les grands de la distribution refuseront toujours de diffuser des films au contenu politique gênant pour le pouvoir - question de bon voisinage entre le pouvoir et l'argent. Donc, pas d'expression politique réelle dans le secteur de grande diffusion. Sauf, bien sûr, des critiques de ce qui se passe à l'extérieur de nos frontières. [...] Vous ferez des films, librement peut-être, sans ennuis de la censure, c'est possible - mais personne ne les verra.» ${ }^{3}$

Et Vautier d'en conclure: «je me suis rendu compte en peu d'années que tout ce que m’avait annoncé le ‘ grand commis de l'Etat`se mettait en place, pour verrouiller l'expression des réalités par l'image et le son ${ }^{4}$. C'est ce qui s'est semble-t-il passé en France pour bon nombre de ses
2 Vautier, op.cit., p. 5.

4 Id., p. 7.
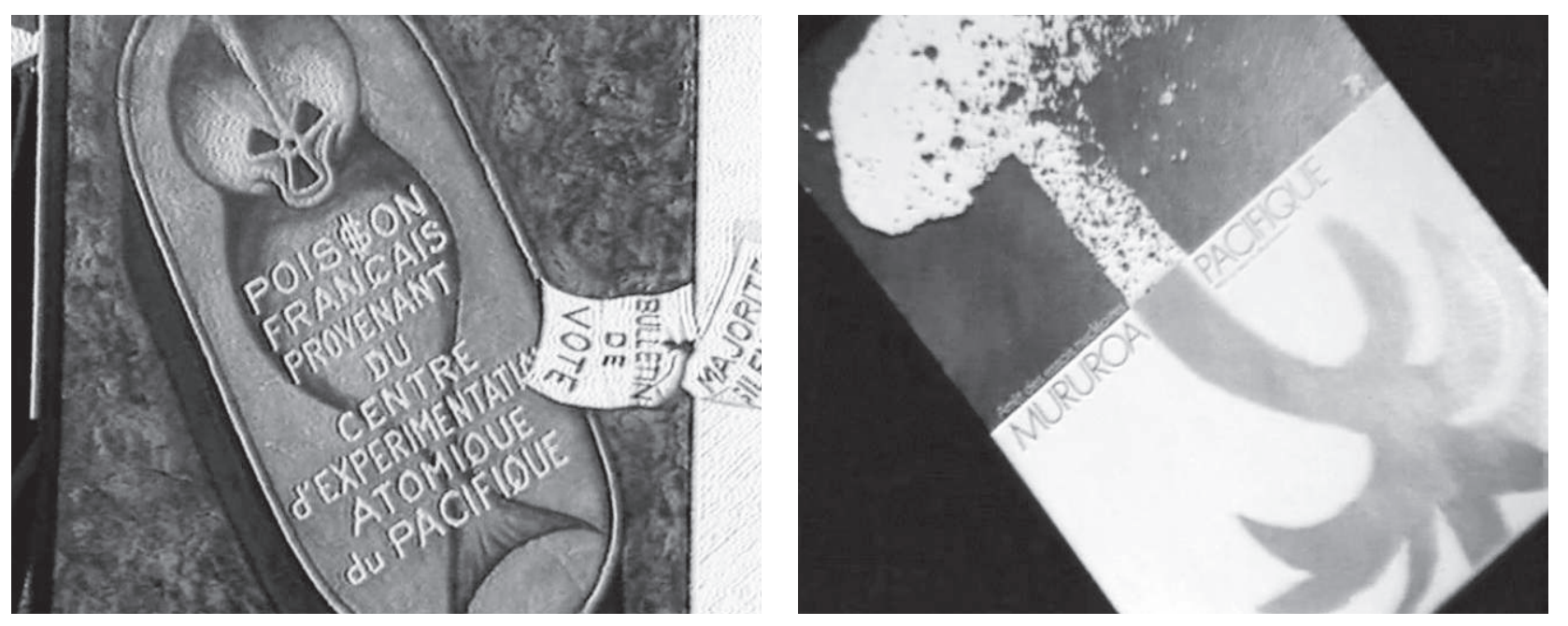
5 Le journal télévisé d'Antenne 2 du I5 juin I986 peut-être visionné en ligne: www.dailymotion.com/video/xfbbnb_ ja2-2oh-emission-du-r5-juin-r986_news. Notons que le court reportage se clôt sur ces affirmations: «Bref, une journée qui veut montrer que la lutte pour la paix est la même à l'Ouest qu'à l'Est. Une idée pourtant qui est loin de rassembler tous ceux qui disent se battre pour la paix» sans pour autant donner de détails sur les divisions que cette remarque insinue, ni sur le véritable combat de ceux qui «disent» œuvrer pour la paix, comme s'il s'agissait là d'une manœuvre populiste qui masquait d'autres types d'affrontement. Le message télévisuel tend donc vraisemblablement à mettre en évidence le fait que la paix est un prétexte qui divise.

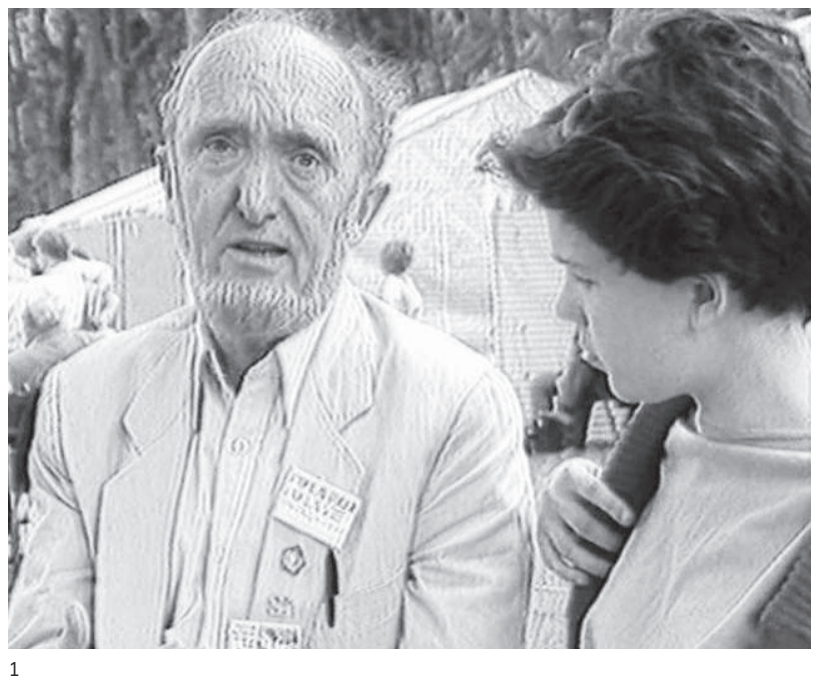

films, bien que le problème ne se cantonne pas là, car la censure ne s'applique pas qu'aux œuvres audiovisuelles. A ce propos, Albert Jacquard ( fig. I), généticien français et auteur de célèbres ouvrages de vulgarisation, interviewé par René Vautier à l'occasion d'une grande manifestation pacifique le 5 juin 1986 dans le cadre du film Paris pour la paix, peut déclarer:

«nous sommes quelques centaines de milliers, mais personne n'en a parlé. Il n'y a pas eu une minute à la radio, une minute à la télévision, pour annoncer que l'événement avait lieu. Et bien est-ce que cette absence même d'information ne constitue pas en soi un événement?»

Le silence médiatique semble ainsi confirmer une nouvelle fois, et plus largement, la prophétie gouvernementale évoquée plus haut. Ce cas est d'autant plus étonnant au vu de l'ampleur populaire de l'événement - précisons toutefois qu’il a tout de même été traité dans le journal télévisé du soir même sur Antenne 2, chiffrant alors le nombre de manifestants à 30000 personnes ${ }^{5}$. Malgré la mention succincte de la manifestation sur une chaîne publique française, c'est bien l'absence d'annonce, de publicité, que Jacquard regrette et condamne. Paris pour la paix, dont les événements se déroulent le jour de la manifestation, 
est le premier film de René Vautier qui se positionne clairement contre l'utilisation du nucléaire, civile ou militaire. Son récit s'articule autour du témoignage d'une jeune fille, venue participer à la manifestation et se muant en journaliste durant la journée. Elle assistera ensuite, dans un cadre virtuel, à une simulation d'attaque nucléaire sur Paris, incarnant ainsi un double du spectateur, elle-même face à un écran sur lequel des images constituées d'effets spéciaux sont projetées (fig. 2-3). Ce dédoublement didactique du spectateur, censé renforcer et préciser l'effet des images (l'attitude à adopter n'étant pas celle d'un individu fasciné par l'aspect grandiose ou purement spectaculaire des explosions), est certes assez naïvement mis en place, mais le dispositif permet au film de modéliser et chiffrer les conséquences d'une attaque nucléaire à l'échelle de la ville de Paris. Paris pour la paix est ainsi composé de témoignages de manifestants, qu'il s'agisse de célébrités ou d'anonymes, formant de la sorte une captation de l'événement (concerts, foule, banderoles en tout genre), auxquels s'ajoutent des séquences de simulation de bombardements et des réactions en chaîne qui s'ensuivraient. Une alternance est ainsi posée entre un régime de témoignages de type purement documentaire et un mode plus fictionnel, voire d'anticipation, qui rappelle The War Game (Peter Watkins, Grande-Bretagne, I965). Mais alors que

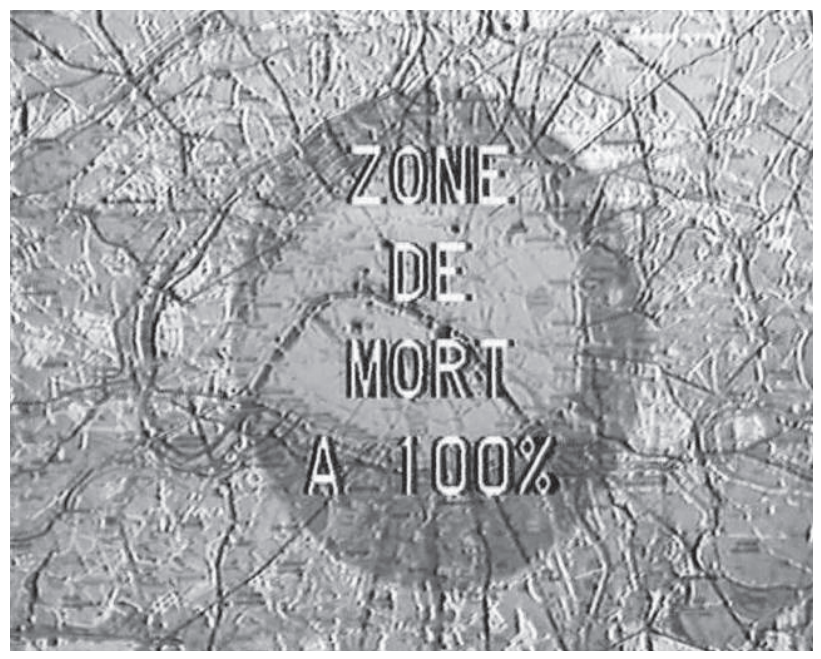

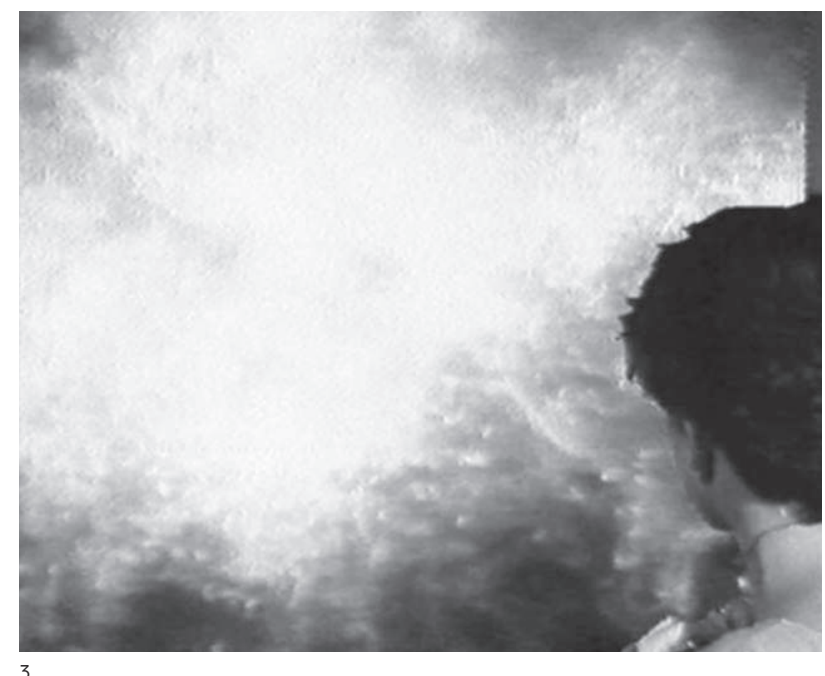


6 Une version du film, datée de i99i, est disponible en ligne sur https://vimeo. $\mathrm{com} / 34526990$. le film de Watkins tentait de brouiller les frontières entre fiction et documentaire, Vautier distingue clairement les deux modes, notamment grâce à l'utilisation de sous-titres explicatifs. La rencontre des deux films se fait au niveau du message alarmiste, qui tente de préciser les différents impacts - tant écologiques, physiques que psychologiques - d'une attaque nucléaire.

Le deuxième film de Vautier traitant du nucléaire, Mission Pacifique ${ }^{6}$, est plus spécifiquement consacré aux essais nucléaires français dans le Pacifique, principalement effectués dans l'atoll de Mururoa, au sein du Centre d'Expérimentation du Pacifique (CEP). Tourné en I995 alors que le nouveau Président de la République française, Jacques Chirac, vient d'être élu, et que ce dernier a ordonné la reprise des essais, il dépeint l'opposition que cela suscite, tant du côté français ou néo-zélandais qu’international, notamment par le biais d'organisations telles que Greenpeace ou le «Mouvement de la Paix» (ce dernier étant le producteur du film). On y suit sa délégation, aux côtés du «personnage principal» du film, Monseigneur Gaillot, évêque d'Evreux. Il est l'interlocuteur privilégié, à chaque étape de l'itinéraire mené par le groupe, et fait figure d'ambassadeur du mouvement, tout en se présentant comme simple individu français, en partie responsable des activités de l'Etat. Ce périple permet de donner la parole aux exilés des atolls, aux habitants de Tahiti et finalement aux Néo-Zélandais, atterrés par l'attitude de la France qui mène des essais loin de sa métropole, tout en affirmant pourtant quaucun risque n'est encouru pour la population locale. Le désarroi des Océaniens est entre autres nourri par «l'affaire du Rainbow Warrior». Le film revient sur les circonstances de la destruction criminelle par les services de renseignement français (la DGSE) du navire affrété par Greenpeace, dix ans auparavant, le ro juillet 1985 , et sur la mort d'un de ses occupants. Une reconstitution est ainsi greffée au documentaire, sur laquelle la voix over de Vautier qualifie cet événement «[d’]un acte terroriste impardonnable. Un acte terroriste commis au nom de la France, en notre nom à nous, gens de France». La fin de son commentaire apparaît presque comme un slogan, celui-ci revenant à plusieurs reprises dans d'autres films du réalisateur (Afrique 50; Marée noire, colère rouge). Et de poursuivre: «Nous n'avons pas le droit, nous Français, d'oublier le visage de ce photographe portugais mort pour avoir voulu mettre ses images au service de la paix. Après avoir nié, le gouvernement français reconnaît au niveau 
le plus élevé sa responsabilité.» Suit finalement l'aveu officiel, en anglais, du premier ministre de François Mitterrand, Laurent Fabius: «La vérité est cruelle, mais il faut que tout soit clairement et totalement exposé. Ce sont de agents de la DGSE qui ont coulé le Rainbow Warrior et ils agissaient sur ordre [notre traduction]». Le ministre faisant suite à Fabius, sous la même présidence, est Jacques Chirac (fig. 4). Voici les propos qu'il tient face à un journaliste néo-zélandais qui lui demande son avis sur l'événement:

[Chirac] I don't want to talk anymore about that.

[Journaliste] But How can you justify calling the Rainbow Warrior almost heroes and posing sanctions on New Zealand?

[Chirac] Well, I never said anything like that. I said this is, as we say in French: a page which is turned. And I don't want to talk anymore about it because I want to forget about that.

Remarquons la formule presque tautologique du futur Président de la République, signalant une gêne très sûrement coupable. Finalement, la voix over de Vautier poursuit:

«Une page tournée, une chose qu'il faut oublier? Ce n'est pas l'avis de tous ces gens, qu'en une ronde de réunions à travers Nouvelle-Zélande

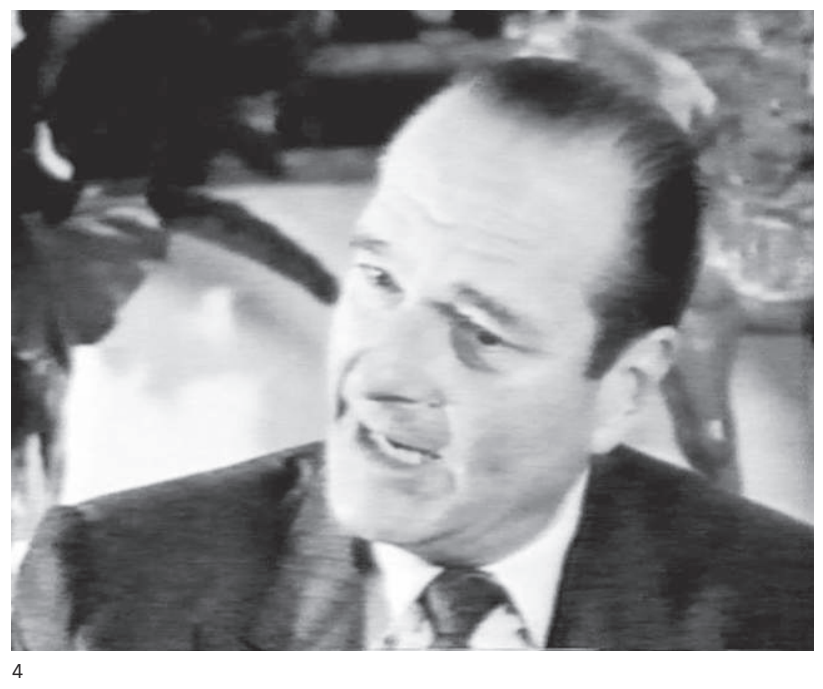



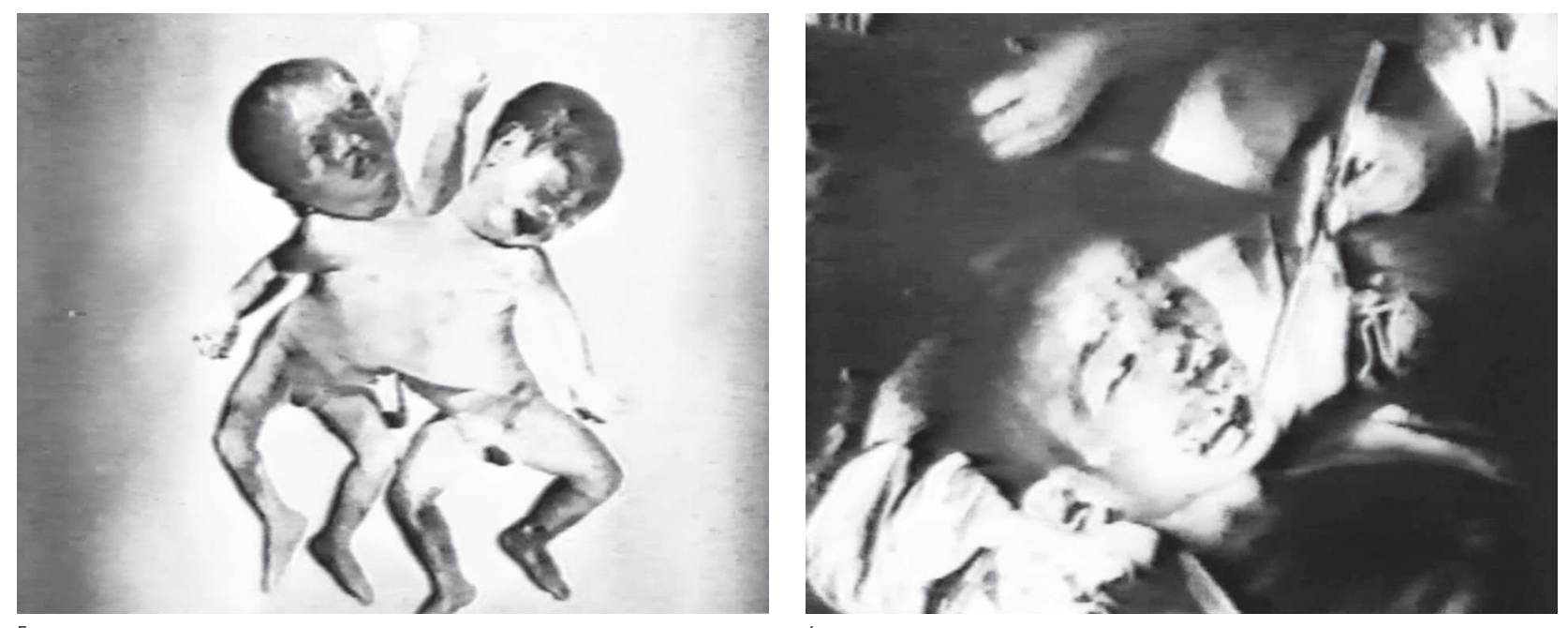

et Australie, la délégation du Mouvement de la paix a rencontrés en cet automne quatre-vingt-neuf. Pour eux, la liaison entre l'acte terroriste contre le Rainbow Warior et les essais nucléaires était évidente, incontournable, et la seule réponse positive de la France aurait dû être pour tous l'arrêt total de ces essais nucléaires.»

Le film souligne qu'une telle action de la part d'un gouvernement trahit une volonté de dissimulation des effets des essais sur l'environnement (au sens large), et regrette que tout cela n'ait pas abouti à une prise de conscience plus générale et corrélativement débouché sur un mouvement de désinvestissement, plutôt que sur la reprise de la politique nucléaire dans le Pacifique. Désirant faire progresser l'opinion et ainsi modifier le cours des événements, le réalisateur tente de susciter une prise de conscience auprès des populations locales. C'est par le biais de témoignages, réalisés par Vautier et par $M^{\text {gr }}$ Gaillot, que l'attitude du gouvernement français est dénoncée: récit désabusé d'un descendant de soldat néo-zélandais (force alliée à la France) mobilisé lors de la Première Guerre mondiale et ayant succombé aux attaques de gaz, évocation de l’image de la culture française à l'étranger entachée par les essais et le dynamitage du bateau de Greenpeace, etc. 
Troisième film de l'ensemble, Hirochirac 1995 est largement constitué d'autocitations, tirées des deux précédents films, ainsi que d'images d'archives télévisuelles. Vautier profite d'un double événement de cette année 1995 (condensé dans le mot-valise du titre), à savoir les célébrations du cinquantenaire des attaques sur Hiroshima et Nagasaki ainsi que la reprise des essais nucléaires par Jacques Chirac, fraîchement élu à la tête de la République. Ce n'est donc pas étonnant si Vautier, accompagné d’une délégation française au Japon, n’y rencontre ni caméras ni officiels de l'Etat, les dates étant peu propices à un message qui ne serait pas embarrassant, voire contradictoire (les Japonais célèbrent les effets de la bombe nucléaire tandis que la France relance ses essais). Vautier en rapporte des images choquantes (fig. 5-6), dont certaines d'archive, et témoigne:

«Première promenade sur les magnifiques esplanades du parc marquant le centre-ville rasé il y a cinquante ans par la bombe. Nous ne savions pas encore que, dans cette sorte de Ria bretonne, 9000 enfants se sont jetés le 6 août 45, pour apaiser les brûlures de la bombe. 9000 enfants sont morts dans cette eau calme. [...] Nous ne savions pas que ces ombrelles que les familles laissent glisser au fil de l'eau sont chargées de messages pour les petits morts (fig. 7). Tout cela et bien d'autres

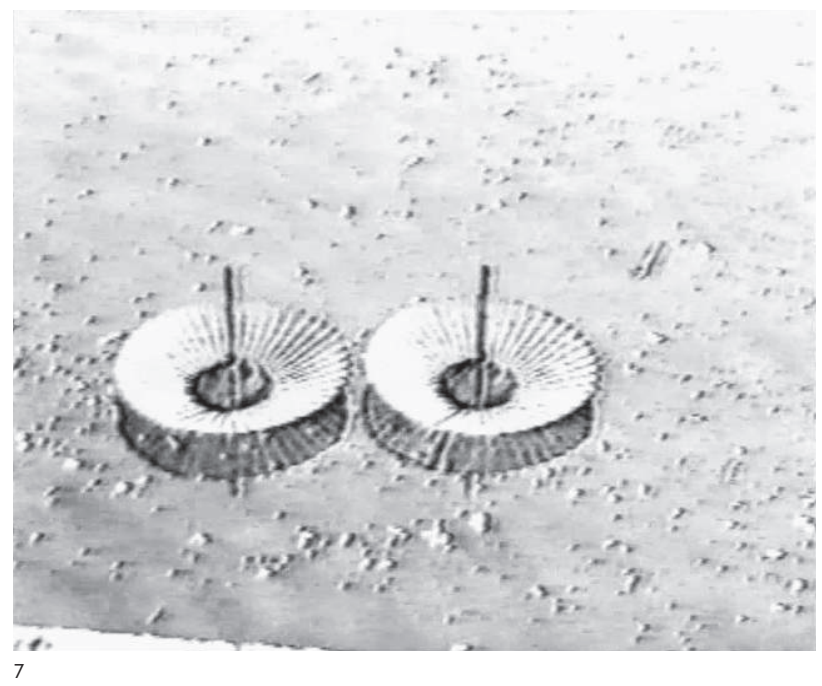


7 Il a fallu attendre la déclassement de ces documents, dont l'article paru dans Le Parisien du I4 février 20I4 «Le document choc sur la bombe A en Algérie» de Sébastien Ramnoux se fait l'écho, pour que le grand public prenne connaissance de l'ampleur de ces essais et de leurs conséquences.

8 Agnès Rougier, «Mise en cause des essais nucléaires dans les maladies des vétérans», en ligne sur le site de Radio France International: www.rfi.fr/france/ 20I207I7-mise-cause-essais-nucleairesmaladies-veterans, dernière consultation le I5 juin 2015 .



choses, nous allions l'apprendre peu à peu et d'abord en visitant le musée d’Hiroshima.»

Après le Japon, c’est au Kazakhstan que les images nous mènent, où un accident trois fois plus grave, nous apprend-on, que Tchernobyl a eu lieu, et où des enfants en nombre meurent de leucémie. Puis c'est au tour du Sahara algérien, où des essais eurent lieu durant la guerre d’Algérie - on nous déclare alors qu'une loi française interdit de montrer les images tournées durant la guerre d’Algérie. Relevons tout de même qu'entre i960 et 1966, I7 essais nucléaires ont été effectués dans le Sahara et que ce n'est qu'en 20I3 que «la carte» des essais nucléaires en Algérie a été déclassifiée de son statut de «secret défense» ${ }^{7}$. L’année précédente, en 20I2, on pouvait relever que «[pour] la première fois, un expert désigné à la demande de la justice établit un lien probable entre les retombées radioactives et un certain nombre de maladies comme des cancers et des leucémies, constatées chez les militaires ayant participé aux essais [en Polynésie et dans le Sahara]» ${ }^{8}$. Notons finalement qu'en février 20I0 un colloque international sur les effets des essais atomiques français au Sahara s’est tenu à Alger, sans que les participants aient pour autant eu accès aux archives les concernant. 
Le film s'achève sur une dernière manifestation antinucléaire à Paris, où Vautier doit faire face à un CRS qui prétend qu'il n'a pas le droit de filmer sans autorisation. La séquence se clôt sur un plan fixe (fig. 8), la caméra montrant l'arrestation et l'embarquement de manifestants par les forces de l'ordre, après l'altercation entre Vautier et l'agent. La voix over de Vautier se fait une nouvelle fois entendre:

«Pourquoi terminer sur ces images? Pour le clin d'oil bien sûr, mais aussi parce que tout ce que dit le commandant de CRS est entièrement faux. Il l'invente au fur et à mesure; caché sous son uniforme, il crée une nouvelle loi. Il est parfaitement permis en France de filmer. Simplement, lorsqu'un pouvoir va à l'encontre d'une volonté populaire, les représentants de l'ordre sont amenés à sortir de la loi, forts de l'uniforme qui leur assure l'impunité. A nous, tous ensemble, de faire respecter la loi en défendant la paix, dont nous sommes en fait les vrais gardiens.»

L'année 2015 célèbre tristement le $70^{\mathrm{e}}$ anniversaire des attaques sur Hiroshima et Nagasaki, tandis qu'au même moment l'utilisation de réacteurs nucléaires redémarre au Japon (ils étaient tous à l'arrêt depuis deux ans) et que seules quelques centaines de personnes ont manifesté contre la reprise des activités nucléaires civiles dans le pays. Notons qu’un de ces manifestants était l'ancien premier ministre japonais Naoto Kan, à la tête de l'Etat lors de l'accident nucléaire de Fukushima, le iı mars 20 II. Quelque temps après la catastrophe, ils étaient plus de i70 ooo à manifester contre le nucléaire. Aujourd'hui, ils ne sont plus qu'une centaine. Suivant les propos, renouvelés, de René Vautier, la présentation de ce corpus de films appelle donc à la vigilance. 